\title{
Mitonuclear co-introgression opposes genetic differentiation between phenotypically divergent songbirds
}

$$
\text { Ellen Nikelski1, *, Alexander S. Rubtsov², and Darren Irwin }{ }^{1}
$$

${ }^{1}$ Department of Zoology, and Biodiversity Research Centre, 6270 University Blvd., University of British Columbia, Vancouver, BC, Canada

$9 \quad{ }^{*}$ Present address: Department of Ecology and Evolutionary Biology, University of Toronto, Toronto, ON, Canada

11 Running Title:

12 Co-introgression opposes differentiation

14 Corresponding Author:

15 Ellen Nikelski, Department of Ecology and Evolutionary Biology, University of Toronto,

16 Toronto, ON, Canada. Email: ellen.nikelski@mail.utoronto.ca

Keywords:

19 mitonuclear co-introgression, mtDNA, mitonuclear gene, genetic differentiation, chromosome Z,

20 Aves 


\section{Abstract}

33 Comparisons of genomic variation among closely related species often show more differentiation

34 in mitochondrial DNA (mtDNA) and sex chromosomes than in autosomes, a pattern expected

35 due to the relative effective population sizes of these genomic components. Differential

36 introgression can cause some species pairs to deviate dramatically from this pattern. The

37 yellowhammer (Emberiza citrinella) and the pine bunting (E. leucocephalos) are hybridizing

38 avian sister species that differ greatly in appearance but show no mtDNA differentiation. This

39 discordance might be explained by mtDNA introgression - a process that can select for co-

40 introgression at nuclear genes with mitochondrial functions (mitonuclear genes). We investigated

41 genome-wide nuclear differentiation between yellowhammers and pine buntings and compared it

42 to what was seen previously in the mitochondrial genome. We found clear nuclear differentiation

43 that was highly heterogeneous across the genome, with a particularly wide differentiation peak

44 on the sex chromosome Z. We further tested for preferential introgression of mitonuclear genes

45 and detected evidence for such biased introgression in yellowhammers. Mitonuclear co-

46 introgression can remove post-zygotic incompatibilities between species and may contribute to

47 the continued hybridization between yellowhammers and pine buntings despite their clear

48 morphological and genetic differences. As such, our results highlight the potential ramifications

49 of co-introgression in species evolution.

\section{Introduction}

Evolution in eukaryotes is shaped by changes in multiple genomic components that differ

53 in their modes of inheritance: mitochondrial DNA (mtDNA) is usually inherited through the 
54 matrilineal line, autosomes are inherited through both parental lines and sex chromosomes are

55 inherited differentially depending on the sex of both parent and offspring (Avise, 2000). There is

56 often much variation among these genomic components in the degree of genetic differentiation

57 between related populations or species (reviewed in Coyne \& Orr, 2004; reviewed in Price,

58 2008), suggesting that their dynamics differ during the process of speciation of a single species

59 into two or more. This variation can arise through differences in both the rate at which specific

60 DNA sequences evolve and the degree to which different components contribute towards genetic

61 incompatibilities that reduce gene flow between populations. A common pattern observed

62 between speciating taxa is clear differentiation in mtDNA (eg. Hebert et al. 2004; Kerr et al.

63 2007), moderate differentiation in sex chromosomes (eg. Thornton \& Long, 2002; Borge et al.

64 2005; Lu \& Wu, 2005; Harr, 2006; Ruegg et al. 2014; Sackton et al. 2014), and comparatively

65 modest differentiation across autosomes (Harr, 2006; Nadeau et al. 2012; Irwin et al. 2018).

66 Measures of mtDNA differentiation are often used to identify and classify genetically

67 distinct populations (eg. Hebert et al. 2004; Kerr et al. 2007) and to infer their histories (Moore,

68 1995; Zink \& Barrowclough, 2008). Due to its uniparental inheritance, mtDNA has one quarter

69 the effective population size and coalescence time of autosomal nuclear DNA (Moore, 1995).

70 This characteristic combined with mtDNA's relatively high mutation rate (Lynch et al. 2006)

71 mean that genetic differences arise and fix relatively quickly, creating patterns of clear mtDNA

72 differentiation between recently diverged populations.

73 Sex chromosomes are another genomic region that often shows higher between-

74 population genetic differentiation compared to autosomes between speciating taxa, in both $\mathrm{Z} / \mathrm{W}$

75 (Borge et al. 2005; Ruegg et al. 2014; Sackton et al. 2014) and X/Y systems (Thorton \& Long,

76 2002; Lu \& Wu, 2005; Harr, 2006). To explain this “faster Z/X effect," researchers have noted 
77 that, because beneficial recessive mutations on the $\mathrm{Z}$ or $\mathrm{X}$ chromosome are immediately exposed

78 to selective forces in the heterogametic sex, fixation of these mutations should proceed faster

79 than if the mutations appeared on autosomes (reviewed in Meisel \& Connallon, 2013; Irwin,

80 2018). Also contributing to genetic differentiation on the $Z$ and $X$ chromosomes are the lower

81 effective population sizes of these chromosomes compared to autosomes (Mank et al. 2010;

82 reviewed in Irwin, 2018). A lower effective population size allows for the fixation of a greater

83 number of slightly deleterious mutations due to less effective purifying selection and a larger role

84 of genetic drift. It is likely that both forces - the faster $\mathrm{Z} / \mathrm{X}$ effect and less effective purifying

85 selection - contribute to the moderate amount of genetic differentiation seen between the sex

86 chromosomes of diverging taxa (Thorton \& Long, 2002; Borge et al. 2005; Lu \& Wu, 2005;

87 Harr, 2006; Ruegg et al. 2014; Sackton et al. 2014).

Differentiation across autosomes, which tends to be lower than on mtDNA and sex

89 chromosomes, can be highly heterogeneous. In fact, many researchers report "islands of

90 differentiation" on autosomes where peaks of high relative differentiation are found against a

91 background of low relative differentiation (e.g., Harr, 2006; Nadeau et al. 2012; Hejase et al.

92 2020). Explanations for these "islands" usually invoke reduced gene flow (reviewed in Wu,

93 2001) and/or repeated bouts of selection (Cruickshank and Hahn, 2014; Irwin et al. 2018). In the

94 former scenario, differentiation peaks are hypothesized to house the loci responsible for

95 reproductive barriers between interacting taxa and, as a result, they are resistant to the gene flow

96 that homogenizes the rest of the nuclear genome. In contrast, explanations invoking repeated

97 selection hypothesize that differentiation islands are areas of the genome that experienced

98 repeated reductions in genetic diversity as a result of selection or selective sweeps in both

99 ancestral and daughter populations. 
Despite the general patterns of differentiation discussed above, an increasing number of

101 studies report remarkably low differentiation between populations at what are normally highly

102 divergent genetic components when compared to other genetic regions or observable phenotypes

103 (e.g., Irwin et al. 2009; Yannic et al. 2010; Bryson et al. 2012). In a number of cases, mtDNA

104 shows dramatically low differentiation when compared to differentiation of the nuclear genome,

105 a pattern referred to as "mitonuclear discordance" (reviewed in Toews \& Brelsford, 2012).

106 Discordance between marker types may be explained by hybridization and introgression between

107 populations, perhaps due to a selective advantage of the introgressing genetic region. For

108 example, Hulsey et al. (2016) documented low mtDNA differentiation-likely due to

109 introgression - and clear differentiation in nuclear DNA (nucDNA) between two hybridizing

110 cichlid species (Hulsey \& García de León, 2013). The researchers further reported high mtDNA

111 differentiation between isolated populations of cichlids at genetic sites associated with thermal

112 tolerance and a significant correlation between mtDNA divergence and water temperature

113 (Hulsey et al. 2016). Altogether, these results suggest that mtDNA introgression produced the

114 discordance seen between marker types and that this outcome was potentially driven by adaptive

115 selection for tolerance of extreme water temperatures.

The hypothesis of adaptive introgression increases in complexity if we consider the

117 potential for coevolution between genomic components. Research investigating coevolution

118 between mitochondrial and nuclear genomes is relatively novel as mtDNA was often treated as a

119 neutral marker in past evolutionary research (Avise, 2000). Nevertheless, recent empirical and

120 theoretical work has provided greater context regarding how mitonuclear coevolution may

121 influence the progression of differentiation and speciation between taxa (Hill, 2019). 
Mitonuclear ecology is the study of how forces acting on the mitochondrial and nuclear

123 genomes interact to influence ecological and evolutionary processes (Hill, 2019). Best known for

124 aerobic respiration, the mitochondrion is the site of the electron transport chain (ETC)

125 responsible for oxidative phosphorylation in eukaryotic organisms (reviewed in Ernster \&

126 Schatz, 1981). Due to the mitochondrial genome's reduced size of about 37 genes, proper

127 functioning of the ETC as well as transcription, translation and replication of mtDNA is reliant

128 on about 1500 proteins encoded by mitonuclear genes within the nuclear genome (Calvo \&

129 Mootha, 2010; Lotz et al. 2014). This interplay between mtDNA and nucDNA implies

130 coevolution between the two genomes such that changes in one places selection on the other for

131 compensatory changes that reduce genetic incompatibility and maintain mitochondrial function

132 (Gershoni et al. 2009; Burton \& Barreto, 2012; Hill, 2019). Secondary contact and hybridization

133 between differentiated populations can result in hybrid breakdown due to mismatches between

134 coevolved combinations of mtDNA and mitonuclear genes, contributing to reproductive isolation

135 and eventual speciation of the groups. Yet, introgression of an adaptive mitochondrial haplotype

136 from one population into another could select for similar introgression of compatible alleles at

137 mitonuclear genes (eg. Beck et al. 2015; Morales et al. 2018; Wang et al. 2021). Such co-

138 introgression would act as a homogenizing force and decrease genetic differentiation between

139 populations in both the mitochondrial and nuclear genomes.

The potential for introgression between speciating populations depends on the degree to

141 which they are in geographic contact during their period of differentiation. In many cases,

142 closely related species occur in allopatry, but in others, taxa meet in areas of contact where they

143 may interbreed (reviewed in Coyne \& Orr, 2004; reviewed in Price, 2008). Successful

144 hybridization and backcrossing allow for the introduction of genetic variation from one 
145 population into the other and for adaptive introgression or co-introgression between taxa at

146 particular genomic regions which can create the discordant differentiation patterns noted in many

147 systems (reviewed in Toews \& Brelsford, 2012). To look for evidence of adaptive co-

148 introgression, we must therefore examine systems where two species are or have hybridized

149 previously and where different genomic components show discordant patterns of genetic

150 differentiation.

An example of one such system includes the yellowhammer (Passeriformes:

152 Emberizidae: Emberiza citrinella) and the pine bunting (Emberiza leucocephalos) —an avian

153 species pair thought to have diverged during the Pleistocene glaciations when they were

154 separated on either side of Eurasia by an area of unsuitable habitat (Irwin et al. 2009). These taxa

155 are highly divergent in plumage and moderately divergent in song and ecology (Panov et al.

156 2003; Rubtsov \& Tarasov, 2017). Despite their differences, yellowhammers and pine buntings

157 hybridize extensively in a large contact zone in central and western Siberia (Panov et al. 2003;

158 2007; Rubtsov, 2007; Irwin et al. 2009; Rubtsov \& Tarasov, 2017). Genomic work has identified

159 mitonuclear discordance between allopatric yellowhammers and pine buntings (Irwin et al. 2009)

160 as they possess almost no mtDNA divergence but show moderate differentiation in nuclear

161 AFLP (Amplified Fragment Length Polymorphism) markers. To explain these results, Irwin et

162 al. (2009) suggested that mtDNA may have introgressed from one species into the other during a

163 previous selective sweep, and this hypothesis was supported by several statistical tests performed

164 on the mtDNA haplotype network. Such mtDNA introgression could select for co-introgression

165 at mitonuclear genes if sizeable genetic differentiation had developed between yellowhammers

166 and pine buntings (Sloan et al. 2017; Hill, 2019; Wang et al. 2021) which is implied by their

167 divergent behaviour, ecology and appearance. Mitonuclear co-introgression and the resulting 
168 lack of mitonuclear incompatibility could facilitate the continued hybridization seen between

169 yellowhammers and pine buntings and prevent the build-up of reproductive isolation and full

170 speciation between these taxa (Hill, 2019).

171 Here we present the first largescale comparison of DNA sequence variation across the

172 nuclear genomes of allopatric yellowhammers and pine buntings. We address several key

173 questions regarding genetic differentiation in this system. First, does nucDNA differentiation

174 between yellowhammers and pine buntings resemble that of mtDNA (virtually none), that of

175 plumage phenotype (very strong differentiation), or something in between? The earlier AFLP

176 results suggested clear differentiation of nuclear markers between groups (Irwin et al. 2009), but

177 those results were not based on actual DNA sequences. Substantial nuclear differentiation would

178 provide stronger support for the hypothesis that there has been mitochondrial introgression and

179 replacement. Second, what is the structure of differentiation across the nuclear genome? The

180 degree of heterogeneity in differentiation across the genome can be used to test whether adaptive

181 introgression may have occurred in this system, and whether certain regions of the genome (e.g.,

182 the sex chromosomes) may be especially important during divergence. Third, is there an over-

183 representation of known mitonuclear genes within putatively introgressing nucDNA regions-a

184 pattern consistent with mitonuclear co-introgression? Evidence of mitonuclear co-introgression

185 and the resulting loss of mitonuclear incompatibility could offer a possible explanation for the

186 extensive hybridization seen between yellowhammers and pine buntings as well as implicate this

187 process as a homogenizing force that counters divergence between these groups. 


\section{Sampling}

We included 109 blood and tissue samples in this study: 53 phenotypic yellowhammers,

19242 phenotypic pine buntings, and 14 other members of Emberizidae (one Emberiza aureola

193 [yellow-breasted bunting], one Emberiza calandra [corn bunting], one Emberiza cioides

194 [meadow bunting], one Emberiza hortulana [ortolan bunting], four Emberiza stewarti [white-

195 capped bunting], and six Emberiza cirlus [cirl bunting]) to put variation between yellowhammers

196 and pine buntings into a deeper phylogenetic context (Figure 1A; Table 1; Supplementary Table

197 1). A total of 91 samples were included in the AFLP analysis of Irwin et al. (2009) while 18

198 samples were examined for the first time as part of the present research.

When possible, body measurements and photographs were taken of live birds or museum

skins. Yellowhammer and pine bunting males were also scored phenotypically and sorted into

201 phenotypic classes based on the protocols presented in Panov et al. (2003) and Rubtsov \&

202 Tarasov (2017). Briefly, each male received a score from 0-7 for background plumage colour,

203 the amount of chestnut plumage (vs. yellow or white) at the brow and the amount of chestnut

204 plumage (vs. yellow or white) at the throat. For background colour, birds were assessed on the

205 strength of yellow - ranging from bright yellow to pure white - in head and body plumage that

206 did not show brown or black streaking. Phenotypic scores of 0 are consistent with a

207 phenotypically pure yellowhammer and scores of 7 are consistent with a phenotypically pure

208 pine bunting. Phenotypic classes included: pure citrinella (PC), almost citrinella (SC), citrinella

209 hybrid (CH), yellow hybrid (YH), white hybrid (WH), leucocephalos hybrid (LH), almost

210 leucocephalos (SL) and pure leucocephalos (PL) (Rubtsov \& Tarasov, 2017). Unless stated

211 otherwise, any SC and SL individuals that appeared in the allopatric zones were grouped 
212 together with PC and PL individuals respectively and treated as phenotypic yellowhammers and

213 phenotypic pine buntings in subsequent analyses (Figure 1B).

\section{DNA extraction and genotyping-by-sequencing}

DNA was extracted from samples using a standard phenol-chloroform method. We then

216 divided the DNA samples into four genotyping-by-sequencing (GBS) libraries (Elshire et al.

217 2011). The 109 samples included in this study were sequenced in libraries together with 226

218 yellowhammer, pine bunting and hybrid DNA samples collected near and within the sympatric

219 zone as part of a larger project (Nikelski et al. in prep). The libraries were prepared as per the

220 protocol described by Alcaide et al. (2014) with the modifications specified by Geraldes et al.

221 (2019) except that we maintained a 300-400 bp fragment size during size selection. Paired-end

222 sequencing was completed by Genome Québec using the Illumina HiSeq 4000 system,

223 producing more than 1.2 billion reads, $150 \mathrm{bp}$ in length, across the four GBS libraries.

\section{Genotyping-by-sequencing data filtering}

Processing of GBS reads for the samples analyzed in this study was done in conjunction

226 with reads from the samples included in the larger project mentioned above. We processed the

227 reads following Irwin et al. $(2016 ; 2018)$, as summarized here. Reads were demultiplexed using a

228 custom perl script designed by Baute et al. (2016). Next, reads were trimmed for quality using

229 Trimmomatic version 0.36 (Bolger et al. 2014) with the parameters: TRAILING:3,

230 SLIDINGWINDOW:4:10, MINLEN:30. Trimmed reads were aligned to the zebra finch

231 reference genome (Taeniopygia guttata version 3.2.4; Warren et al. 2010) using the program

232 BWA-MEM (Li \& Durbin, 2009) and a BAM file of this information was created for each

233 individual using the programs Picard (http://broadinstitute.github.io/picard/) and SAMtools (Li et 
234 al. 2009). Then, BAM files were converted into GVCF files using the HaplotypeCaller command 235 as part of GATK version 3.8 (McKenna et al. 2010). We processed the resulting GVCF files in

236 two ways to create 1) a genome-wide "variant site" VCF file containing information only on

237 variant sites, and 2) a series of chromosome-specific "info site" VCF files containing information

238 on both variant and invariant sites with sufficient coverage.

To create the genome-wide "variant site" VCF file, we used the GenotypeGVCFs

command in GATK version 3.8 to identify and isolate single nucleotide polymorphisms (SNPs)

241 among individuals. This command also converted the variant site information into a single VCF

242 file encompassing the entire nuclear genome. Using a combination of VCFtools (Danecek et al.

243 2011) and GATK, we filtered the VCF file to remove indels and non-biallelic SNPs. We also

244 discarded loci with $\mathrm{QD}<2.0, \mathrm{MQ}<40.0, \mathrm{FS}>60.0, \mathrm{SOR}>3.0$, or ReadPosRankSum $<-8.0$.

245 Finally, loci with more than $60 \%$ missing genotypes were removed. The average coverage of

246 variable sites in the resulting VCF file was 16.59.

To convert GVCF files into "info site" VCF files, we similarly employed the

248 GenotypeGVCFs command in GATK with the addition of the -allSites and -L flags to retain

249 invariant sites and split the information into chromosome-specific files. The resulting VCF files

250 were filtered using VCFtools and GATK to remove indels, sites with more than two alleles, sites

251 with more than $60 \%$ missing genomic data, sites with MQ values lower than 20 and sites with

252 heterozygosities greater than $60 \%$ (to avoid potential paralogs). Use of these filters simplified

253 calculations in downstream analyses and ensured that these analyses were restricted to sites with

254 sufficient data. 


\section{Variant site analyses}

The genome-wide "variant site" VCF file was analyzed using modified versions of the R scripts described in Irwin et al. (2018). A total of 374,780 SNPs were identified among allopatric

260 yellowhammers and pine buntings. For each of these SNPs we calculated sample size, allele

261 frequency, and Weir and Cockerham's $F_{\text {ST }}$ (Weir \& Cockerham, 1984). Genetic differentiation

262 between yellowhammers and pine buntings was then visualized using a principal components

263 analysis (PCA) generated with the pca command and the svdImpute method to account for any

264 missing genomic data using the pcaMethods package (Stacklies et al. 2007). The PC1 loadings

265 were also graphed as a Manhattan plot using the package qqman (Turner, 2018). Finally, to

266 examine the spread of variant sites across the genome and identify areas of high differentiation,

267 the $F_{\text {ST }}$ values of 349,807 SNPs were graphed as a Manhattan plot. The remaining SNPs did not

268 possess known genomic locations and, therefore, could not be included in the plot.

\section{Differentiation across the genome}

To thoroughly investigate genomic differentiation between allopatric yellowhammers and

271 pine buntings, we performed further analysis on both variant and invariant loci within "info site"

272 VCF files using R scripts described in Irwin et al. (2018).

We calculated Weir and Cockerham's $F_{\mathrm{ST}}$, between-group nucleotide distance $\left(\pi_{B}\right)$ and

274 within-group nucleotide diversity $\left(\pi_{W}\right)$ for nonoverlapping windows of available sequence data

275 across each chromosome. The first window was positioned at the "start" of each chromosome as

276 described in the zebra finch reference genome (Warren et al. 2010). We used a window size of

$2772000 \mathrm{bp}$ of sequenced data rather than 10,000 bp (as in Irwin et al. 2018), to visualize narrow

278 peaks in relative and absolute differentiation within our dataset. 
We developed a new R script to calculate a Tajima's D value (Tajima, 1989) for each of

280 the $2000 \mathrm{bp}$ windows. Values of Tajima's D were used to identify areas of the genome where

281 patterns of variation in yellowhammer and pine bunting populations deviated from models of

282 neutrality. Significantly negative Tajima's D implies that there are more rare alleles in a

283 population than expected under neutrality, likely because of a selective sweep or population

284 expansion following a bottleneck. Significantly positive Tajima's D suggests that there are fewer

285 rare alleles in a population than expected under neutrality, potentially stemming from balancing

286 selection or rapid population contraction.

\section{Phylogenetic comparison with other Emberizidae species}

We employed whole-genome averages of $\pi_{B}$ between allopatric yellowhammers and

289 allopatric pine buntings as well as among these focal species and six other Emberizidae species

290 (Emberiza aureola, Emberiza calandra, Emberiza cioides, Emberiza cirlus, Emberiza hortulana

291 and Emberiza stewarti) to estimate a phylogeny. A list of average $\pi_{B}$ values for each species pair

292 was converted into a distance matrix and used to create an unrooted neighbour-joining tree. This

293 tree was constructed using the ape package (Paradis \& Schliep, 2019) and the BioNJ algorithm

294 (Gascuel, 1997) with Emberiza aureola set as the outgroup (Alström et al. 2008).

\section{Signals of mitonuclear co-introgression}

To test for signals of mitonuclear gene introgression between allopatric yellowhammers

297 and pine buntings, we compiled a list of mitonuclear genes and a list of $2000 \mathrm{bp}$ putative

298 introgression windows (hereafter referred to as "introgression windows") and then tested for an

299 association between them. We explain these steps in detail below. If mitonuclear genes were

300 found within introgression windows statistically more often than was expected due to chance, it 
would provide support for mitonuclear gene introgression potentially in response to the adaptive mtDNA introgression (Hill, 2019) hypothesized to have occurred in this system (Irwin et al. 2009).

To start, we created a list of mitonuclear genes to analyze for signals of introgression. We chose mitonuclear genes that were protein-coding and whose protein products interacted directly with mtDNA or an immediate product of the mitochondrial genome (i.e. protein or RNA). For these nuclear-encoded genes, any change in mtDNA including those caused by introgression

308 would likely cause selection for co-introgression of compatible alleles (Gershoni et al. 2009;

309 Burton \& Barreto, 2012; Hill, 2019). Mitonuclear genes that met these criteria included those

310 that encode protein subunits of ATP synthase or the first, third and fourth complex of the ETC,

311 assembly and ancillary proteins involved in the formation of the ETC, or proteins that are part of 312 the transcription, translation or DNA replication machinery within the mitochondria. This list of 313 genes was created using information from Figure 2.3 and Table 2.1 in Hill (2019). After

314 removing any genes that were not annotated in the zebra finch reference genome or that lacked a 315 specific location on the reference genome, a total of 134 mitonuclear genes remained for analysis 316 (Supplementary Table 2). Next, we identified introgression windows across the genome as those possessing both a

318 low Tajima's D value and a low $\pi_{B}$ value. Low $\pi_{B}$ indicates high similarity between the

319 nucleotide sequences of two groups as would be expected if mitonuclear gene alleles had

320 introgressed from one taxon into the other. Low Tajima's D suggests a past selective sweep

321 within a population which would also be expected if an adaptive mitonuclear allele had 322 introgressed from a separate taxon and swept throughout the receiving population. For this 323 analysis, our quantitative criteria for an introgression window were a Tajima's D value within 
324 the lowest $5 \%$ of the available windowed values and a $\pi_{B}$ value within the lowest $30 \%$ of the

325 available windowed values. Out of the 7187 windows described across the genome, 244

326 introgression windows were identified for yellowhammers and 222 introgression windows were

327 identified for pine buntings. As well, of the introgression windows identified in yellowhammer

328 and pine bunting populations, 71 were shared between the taxa. It should be noted that sharing of

329 some introgression windows is expected given that the contribution of $\pi_{B}$ to window selection

330 was identical for both taxa (in contrast, Tajima's D was calculated separately for yellowhammers

331 and pine buntings).

Following window classification, we employed a custom R script to determine how many

333 mitonuclear genes occurred within introgression windows. To do this, we identified the genomic

334 centre position of each mitonuclear gene as well as the average genomic position of each of the

335 genomic windows. We then calculated the absolute difference between mitonuclear gene centres

336 and average window positions. Mitonuclear genes were assumed to occur within whatever

337 window minimized this difference. With this information, we were able to determine the number

338 of mitonuclear genes that occurred within the introgression windows of each taxon.

The number of mitonuclear genes within introgression windows were compared to what

340 would be expected if these genes were distributed randomly across the genome using separate

341 two-tailed binomial tests for yellowhammers and pine buntings. Because genes are often not

342 distributed randomly and may appear more densely packed in certain genomic regions

343 (International Chicken Genome Sequencing Consortium, 2004), we also conducted a Fisher's

344 Exact test for both yellowhammers and pine buntings to determine whether the proportion of

345 mitonuclear genes within introgression windows was significantly different from what would be

346 expected based on the total proportion of protein coding genes appearing within these windows. 


\section{Results}

When comparing allopatric yellowhammers and pine buntings, following filtering we

349 identified 374,780 variable SNPs within our "variant site" VCF file and 13,703,455 invariant and

350699,122 variant sites across thirty autosomes and the Z chromosome within our "info site" VCF

351 files. In the latter "info site" files, we designated a total of 7187 genomic windows (of 2000

352 sequenced bp each) across the genome, with each window covering an average distance of about

353139 kilobases.

\section{Phylogenetic comparison with other Emberizidae species}

An unrooted neighbour-joining tree of average $\pi_{B}$ values between yellowhammers, pine

356 buntings and six other Emberizidae species (Figure 2) depicted similar species relationships as

357 were identified previously using mitochondrial markers (Alström et al. 2008; Irwin et al. 2009).

358 As well, similar relative branch lengths between taxa were recovered with the exception of that

359 between yellowhammers and pine buntings. In terms of the relative genetic distance $\left(\pi_{B}\right)$

360 between yellowhammers and pine buntings compared to the distance between each of those and

361 E. stewarti, nuclear genetic distance was 11.4 times greater than mitochondrial genetic distance.

362 This corroborates the presence of mitonuclear discordance between the taxa where nucDNA is

363 much more deeply diverged than mtDNA, supporting the hypothesis of an extended period of

364 divergence between yellowhammers and pine buntings followed by mtDNA introgression.

\section{Overall genetic differentiation}

Based on 374,780 SNPs considered all together, our genome-wide $F_{\text {ST }}$ estimate was

3670.0232 between allopatric yellowhammers and pine buntings. Despite this low average, a PCA

368 based on the same SNP genotypes separated yellowhammers and pine buntings into tight genetic 
clusters (Figure 3). PC1 explained 3.6\% of the variation among individuals while PC2 explained $2.9 \%$ of the variation. Two pine buntings were outliers along $\mathrm{PC} 1$, while the remaining

371 yellowhammers and pine buntings separated into distinct clusters mainly along PC2. Further

372 investigation into these outliers revealed that they were males from the same location. A kinship

373 analysis completed as part of a separate study did not find close kinship between the two pine

374 buntings that could explain their position as outliers (Nikelski et al. in prep). An examination of

375 the PC1 loadings for each of the SNPs revealed that the signal for the PC1 positioning was

376 broadly distributed across the genome, rather than being concentrated in a few specific regions

377 (Supplementary Figure 1). To explore the causes of these outliers, we temporarily removed one

378 of them and re-ran the PCA. This caused the other outlier to fall into the pine bunting cluster, but

379 did reveal a further yellowhammer outlier (Supplementary Figure 2). Removal of this

380 yellowhammer outlier in addition to one member of the pine bunting outlier pair in turn revealed

381 another yellowhammer outlier (Supplementary Figure 3). It is unclear what is responsible for

382 these outliers, but the distinct yellowhammer and pine bunting genetic clusters remained intact in

383 all the PCAs considered.

\section{Differentiation across the genome}

Relative differentiation between allopatric yellowhammers and pine buntings was highly

386 heterogeneous across the nuclear genome with peaks in $F_{\text {ST }}$ seen on most of the larger

387 chromosomes (Figures 4, 5; Supplementary Figure 4). Chromosome Z in particular showed a

388 large peak in $F_{\mathrm{ST}}$ with several SNPs possessing values close to one. In fact, $F_{\mathrm{ST}}$ for the $\mathrm{Z}$

389 chromosome was 0.1246 - more than five times larger than the genome-wide $F_{\mathrm{ST}}$.

391 diversity $\left(\pi_{W}\right)$ were also heterogenous across the genome and comparable to each other in 
magnitude: genome-wide $\pi_{B}=0.0041$; genome-wide $\pi_{W}$ for both taxa $=0.0040$ (Figure 5;

393 Supplementary Figure 4). Because between-group and within-group nucleotide diversity are so

394 intimately related in their evolution and calculation, it is expected that windowed averages of

395 these two statistics will show a highly positive relationship. In fact, most windowed $\pi_{B}$ and $\pi_{W}$

396 averages fell near a 1:1 association line (Figure 6) which is equivalent to no or little

397 differentiation. However, some genomic windows showed much reduced $\pi_{W}$ compared to $\pi_{B}$;

398 these were the windows with high $F_{S T}$. Additionally, we detected a weak negative correlation

399 between the windowed averages of $F_{\mathrm{ST}}$ and $\pi_{B}$ (Spearman's Rank Correlation: $-0.1196, \mathrm{p}<2.2 \times$

$40010^{-16}$; Figure 7) as is hypothesized if peaks in relative differentiation are products of repeated

401 selective events (Cruickshank \& Hahn, 2014; Irwin et al. 2018).

Finally, we found that Tajima's D varied across the genome but was mostly negative

403 (Figure 5; Supplementary Figure 4), consistent with a history of population growth and/or

404 selective sweeps. The average genome-wide Tajima's D was similar between populations: -1.377

405 for yellowhammers and -1.335 for pine buntings.

\section{Signals of mitonuclear co-introgression}

Of the 7187 genomic windows identified across the nuclear genome, we classified 244 as

408 introgression windows within yellowhammers and 222 as introgression windows within pine

409 buntings (Table 2). Average values of $\pi_{B}$ and Tajima's D in yellowhammer introgression

410 windows were 0.0016 and -2.3751 respectively, and 0.0019 and -2.3369 in pine buntings

411 respectively.

Nine mitonuclear genes - $6.7 \%$ of the 134 mitonuclear genes considered — appeared

413 within yellowhammer introgression windows (Table 2). This finding was significant in a two- 
414 tailed binomial test $(\mathrm{p}=0.04952)$ indicating that mitonuclear genes appeared in yellowhammer

415 introgression windows more often than would be expected if they were assigned to windows

416 randomly. However, this finding was not significant in a Fisher's Exact test $(\mathrm{p}=0.1311)$ which

417 takes into account the differing densities of genes across the nuclear genome. Four mitonuclear

418 genes appeared within pine bunting introgression windows $-3.0 \%$ of the genes considered. This

419 result was statistically insignificant in both a two-tailed binomial test $(\mathrm{p}=1)$ and a Fisher's Exact

420 test $(\mathrm{p}=1)$ indicating that mitonuclear genes did not appear in pine bunting introgression

421 windows more often than would be expected due to chance. Overall, the significant signal of

422 introgression in yellowhammers and insignificant signal of introgression in pine buntings could

423 indicate that mitonuclear gene introgression — if it occurred — was biased in the direction of pine

424 buntings into yellowhammers.

The nine mitonuclear genes that appeared within yellowhammer introgression windows

are: APOPT1, COX5A, COX17, MRPL1, MRPL27, MRPL32, NDUFC1, mtSSB, UQCR11

427 (Table 3). Three of these genes encode protein subunits of the mitochondrial ribosome, three

428 encode structural subunits of the ETC, two encode assembly factors of the ETC and one encodes

429 a single-stranded DNA-binding protein involved in mtDNA replication. All putatively

430 introgressed genes appear on separate autosomes except for two genes that appear on

431 chromosome 4. Interestingly, three of the five putatively introgressed genes associated with the

432 ETC are specifically associated with complex IV.

434 ATP5H5I, COX5A, MRPL2 and NDUFB4 (Table 4). All four genes appeared on separate 435 autosomes with three of these genes encoding structural subunits of the ETC and one encoding a 436 protein subunit of the mitochondrial ribosome. The COX5A gene, which encodes a structural 
437 subunit of ETC complex IV, was found in both yellowhammer and pine bunting introgression 438 windows.

\section{Discussion}

Yellowhammers and pine buntings show negligible mtDNA differentiation (Irwin et al.

442 2009) but are well differentiated phenotypically (Panov et al. 2003, Rubtsov \& Tarasov, 2017).

443 Prior to this study, a possible explanation for this pattern was simply rapid phenotypic evolution

444 between two genetically similar sister taxa. Our analysis of nucDNA variation has shown clear

445 separation of allopatric yellowhammers and pine buntings, with strong differentiation peaks in

446 specific parts of the nuclear genome. This result points to these taxa experiencing a long period

447 of separate evolution followed by the hybridization now observed within a large contact zone in

448 western and central Siberia. These results combined with our phylogenetic analysis showing a

449 longer branch length between yellowhammers and pine buntings based on nuclear markers-

450 when compared to a phylogeny based on mtDNA (Irwin et al. 2009)—support recent mtDNA

451 introgression and mitochondrial haplotype replacement in this system likely driven by selection.

452 Our analyses also provided some evidence for the disproportionate introgression of mitonuclear

453 genes between taxa which is consistent with co-introgression discussed in current mitonuclear

454 theory (Hill, 2019).

456 pine buntings (0.0232) was comparable to or sometimes lower than the averages seen between

457 avian subspecies (e.g., subspecies of barn swallow: 0.017-0.026 [Scordato et al. 2017]; myrtle

458 warbler and Audubon's warbler: 0.077-0.106 [Irwin et al. 2018]; yellow- and red-shafted 
northern flickers: 0.098 [Manthey et al. 2016]). This low genome-wide $F_{\text {ST }}$ contrasts with the moderate $F_{\mathrm{ST}}$ averages reported from an analysis of 63 AFLP markers performed on the same

461 populations: 0.078 based on allele frequencies and 0.140 based on band frequencies (Irwin et al.

462 2009). The present study reveals that relative differentiation was highly heterogeneous across the

463 nuclear genome with $F_{\text {ST }}$ peaks on various chromosomes. It is possible that the previous AFLP

464 analysis captured a disproportionate number of loci within these differentiation peaks, thereby

465 inflating $F_{\mathrm{ST}}$ estimates. This comparison highlights the caution that should be taken when

466 interpreting genome-wide averages as highly variable genetic differentiation landscapes can

467 cause large variability in $F_{\text {ST }}$ estimates when they are based on a limited number of loci.

most significantly on the Z chromosome are consistent with the "islands of differentiation" often

470 noted in comparisons of other closely related taxa (Harr, 2006; Nadeau et al. 2012; Irwin et al.

471 2018). In contrast to these islands, the large regions of close similarity in $\pi_{B}$ and $\pi_{W}$ suggests

472 high gene flow between taxa at those regions. This scenario is consistent with the observed

473 extensive hybridization between these taxa (Panov et al. 2003; 2007; Rubtsov, 2007; Rubtsov \&

474 Tarasov, 2017). Nevertheless, the high $F_{\mathrm{ST}}$ regions, those with much reduced $\pi_{W}$ compared to

$475 \pi_{B}$, indicate that they have had low gene flow presumably as a result of divergent selection. It is

476 unlikely that this pattern can be explained by genetic drift over an extended period of geographic

477 separation, as this would result in most genomic regions deviating slightly from $\pi_{B}=\pi_{W}$

478 congruence rather than the observed pattern of extreme heterogeneity. Instead, the pattern

479 suggests that selection acted in a way that lowered $\pi_{W}$ relative to $\pi_{B}$ within "islands of

480 differentiation". Considering that high $F_{\text {ST }}$ regions were associated with relatively low values of

$481 \pi_{B}$, we propose that differentiation islands in this system are most consistent with a model 
invoking repeated bouts of selection that lower nucleotide diversity (Cruickshank \& Hahn, 2014; Irwin et al. 2018). A sweep-before-differentiation model (Irwin et al. 2018) where $F_{\text {ST }}$ peaks are produced by adaptive selective sweeps between populations followed by adaptive selection at the same regions in local populations is particularly in line with the extensive hybridization presently observed between yellowhammers and pine buntings. autosomes is a common observation when comparing closely related species (Borge et al. 2005;

491 been explained by less efficient purifying selection and/or more positive selection on this

492 chromosome (Mank et al. 2010; reviewed in Meisel \& Connallon, 2013; reviewed in Irwin,

493 2018). However, the large regions of the $Z$ chromosome that have $F_{\text {ST }}$ values near zero suggests

494 that additional factors are involved in producing the large and wide island of differentiation on 495 the Z.

497 with an area of low recombination - a region of connected loci that tend to be inherited together,

498 leading to linked selection of nearby loci. Strong divergent selection acting on one SNP would

499 act similarly on all the loci that are linked to it such that a wide, highly divergent genomic block

500 would become fixed and appear as an "island" between taxa (reviewed in Cutter \& Payseur,

501 2013). Areas of low recombination and linkage are often associated with inversion

502 polymorphisms (reviewed in Smukowski \& Noor, 2011) as different orientations of an inversion

503 experience little successful recombination (reviewed in Kirkpatrick, 2010). An exploration of a

504 potential chromosomal inversion within the yellowhammer and pine bunting system is being 
505 investigated as part of a separate study and is supported by preliminary evidence (Nikelski et al.

506 in prep).

While numerous "islands of differentiation" were observed between yellowhammers and

508 pine buntings implying moderate genetic divergence between them, mtDNA introgression has

509 the potential to homogenize their nuclear genomes at mitonuclear genes by selecting for co-

510 introgression of compatible alleles (Beck et al. 2015; Sloan et al. 2017; Morales et al. 2018).

511 Consistent with this idea, a two-tailed binomial test supported preferential introgression of

512 mitonuclear genes in allopatric yellowhammers. Because a comparable signal of introgression

513 was not found in allopatric pine buntings, we suggest that mitonuclear co-introgression could

514 have occurred in the direction of pine buntings into yellowhammers. Yet, these results must be

515 interpreted with caution due to limitations in introgression window identification. Because we

516 employed reduced-representation sequencing that only captures a small portion of the nuclear

517 genome, we have limited resolution in detecting signals of introgression over narrow genomic

518 regions. This may have contributed to the variation we saw in the statistical significance of

519 mitonuclear gene introgression depending on whether gene densities were considered.

520 Nevertheless, the fact that a significant signal of introgression was detected despite limitations is

521 intriguing especially when considered in conjunction with the identities of the mitonuclear genes

522 found in introgression windows.

Three of the mitonuclear genes within yellowhammer introgression windows and three

524 within pine bunting introgression windows encoded structural subunits of the ETC. The ETC is

525 broken into five protein complexes which, through a series of enzymatic reactions, perform

526 oxidative phosphorylation to produce ATP necessary for organism survival (reviewed in Ernster

$527 \&$ Schatz, 1981). Four of the five ETC complexes are made up of subunits encoded by both the 
nuclear and mitochondrial genome (Hill, 2019) and correct fit between differentially encoded

529 subunits is essential for the flow of electrons and protons across the ETC. To put this in

530 perspective, changing even a single amino acid in one subunit can significantly disrupt its ability

531 to interact with other subunits within a ETC complex (eg. Gershoni et al. 2014). Because of the

532 tight interactions within complexes and the consequences of subunit incompatibility,

533 introgression of mtDNA is expected to select for co-introgression of mitonuclear genes encoding

534 ETC structural subunits. Such co-introgression has been detected between differentially adapted

535 populations of eastern yellow robin where mtDNA introgression between populations was

536 followed by similar introgression of mitonuclear genes encoding subunits of complex I (Morales

537 et al. 2018) and between different species of Drosophila where introgression and replacement of

538 the mtDNA of one species during hybridization selected for co-introgression of genes that

539 encode subunits of complex IV (Beck et al. 2015).

Of the ETC complexes, complex IV showed the strongest signal of co-introgression in

541 the yellowhammer and pine bunting system. Three of the genes within yellowhammer

542 introgression windows and one gene within pine bunting introgression windows were associated

543 with this complex. Interestingly, gene COX5A — a structural subunit of complex IV—appeared

544 in both sets of introgression windows. It is unlikely that this gene introgressed in both directions,

545 but it is possible that COX5A adaptively swept in both populations. In this situation, a

546 particularly adaptive allele may have appeared in one species and swept to high frequency before

547 co-introgressing into the other species following mtDNA introgression. The COX5A gene was

548 also one of the subunits that co-introgressed in the Drosophila example discussed above (Beck et

549 al. 2015) lending some support to its particular importance to mitonuclear compatibility. More

550 generally, complex IV is often used as a model for studying mitonuclear interactions due to its 
551 distinctive structure of a core of mtDNA-encoded subunits surrounded by nucDNA-encoded

552 subunits (Saraste, 1999). With such an excess of mitonuclear interactions, incompatibility

553 involving complex IV has been investigated and detected in several systems including within

554 primate xenomitochondrial cybrids (Barrientos et al. 2000) and between different species of

555 Drosophila (Sackton et al. 2003). Furthermore, work by Osada \& Akashi (2012) has provided

556 strong evidence for compensatory co-evolution between mitonuclear genes related to complex

557 IV and mtDNA among primates particularly at interacting amino acids of differentially encoded

558 subunits. Altogether, these results suggest a crucial role for complex IV in mitonuclear co-

559 evolution as it may relate to divergence and speciation between taxa.

Another group of mitonuclear genes that appeared to preferentially introgress within the

561 yellowhammer and pine bunting system were those encoding subunits of the mitoribosome.

562 Unlike the protein-protein interactions occurring within ETC complexes, mitonuclear

563 interactions in the mitoribosome are between nuclear-encoded proteins and mitochondrial-

564 encoded RNA (Hill,. 2019). Protein subunits associate closely with rRNA during the formation

565 of a mitoribosome, but also interact with mRNA and tRNA during the synthesis of mitochondrial

566 proteins (Greber \& Ban, 2016). Currently, research is limited on the extent and importance of

567 interactions between mitoribosomal subunits and mitochondrial RNA. However, the fact that

568 interactions between components are extensive and necessary for the synthesis of the

569 mitochondrial proteins suggests close co-evolution between mtDNA and genes encoding

570 mitoribosomal subunits that could strongly select for mitonuclear co-introgression following

571 mtDNA introgression.

In summary, yellowhammers and pine buntings are sister taxa that are divergent in

573 appearance, song, and ecology (Panov et al. 2003; Rubtsov \& Tarasov, 2017) yet vary in their 
574 genomic differentiation from virtually none (at the mitochondrial genome) to nearly fixed (the

575 differentiation peak on the $\mathrm{Z}$ chromosome). These patterns are best explained by a period of

576 differentiation while geographically separated, followed by hybridization and introgression. We

577 found some evidence of mitonuclear gene introgression in the direction of pine buntings into

578 yellowhammers that is consistent with mitonuclear co-introgression. This occurred preferentially

579 in mitonuclear genes encoding structural components of both the ETC and the mitoribosome,

580 potentially due to mitonuclear incompatability. Mitonuclear incompatibilities are thought to

581 represent an important post-zygotic reproductive barrier between taxa (Gershoni et al. 2009;

582 Burton \& Barreto, 2012; Hill, 2019), meaning mitonuclear co-introgression has the potential to

583 weaken species boundaries. Support for such breakdown may be seen in the extensive and

584 dynamic hybrid zone between yellowhammers and pine buntings (Panov et al. 2003; 2007;

585 Rubtsov, 2007; Rubtsov \& Tarasov, 2017). Further, careful examination of genetic

586 differentiation and reproductive barriers within the yellowhammer and pine bunting hybrid zone

587 would shed light on the possibility of their merging in the future. As well, the inclusion of

588 analyses that compare mtDNA and mitonuclear gene differentiation in a wider range of systems

589 would help to clarify the potentially important role that mitonuclear interactions play in the

590 merging or diverging of species.

593 E. N., D.I., and A.S.R. conceived of this study. A.S.R. collected samples. E. N. and A.S.R.

594 completed molecular techniques. E.G.M.N. conducted data analysis and wrote this manuscript

595 with input from D.I. and A.S.R. 
Acknowledgements:

597 For providing valuable feedback, we thank Dolph Schluter, Eric Taylor, Judith Mank, Elizabeth

598 Natola, Rashika Ranasinghe, Kenneth Askelson, Finola Fogarty, Quinn McCallum, Ana

599 Barreira, Jamie Clarke, Armando Geraldes and Jessica Irwin. For their kindness and support

600 during field work, we thank the Tazeev family and Madelyn Ore. For providing additional

601 samples, we thank The Bell Museum, The Burke Museum of Natural History and Culture, The

602 Field Museum, The State Darwin Museum, The Swedish Museum of Natural History, The

603 Zoological Museum of the Zoological Institute of the Russian Academy of Sciences, the

604 Zoological Museum of the University of Copenhagen and their accompanying personnel. Major

605 research funding was provided by the Natural Sciences and Engineering Research Council of

606 Canada (NSERC CGSM award to E.N, Discovery Grants RGPIN-2017-03919 and RGPAS-

607 2017-507830 awarded to D.I.) and by the Werner and Hildegard Hesse research awards

608 (Research award in Ornithology and Fellowship in Ornithology awarded to E.G.M.N. by the

609 University of British Columbia).

611 Data Accessibility Statement:

612 Raw DNA sequencing reads will be made available on the NBCI Sequence Read Archive upon

613 publication acceptance. Read processing codes, barcodes, genotype data and R codes associated

614 with statistical analyses will be made available on Dryad upon publication acceptance. 
None declared

618

Bibliography

Alcaide, M., Scordato, E. S. C., Price, T. D., \& Irwin, D. E. (2014). Genomic divergence in a ring species complex. Nature, 511(7507), 83-85. doi:10.1038/nature13285

Alström, P., Olsson, U., Lei, F., Wang, H., Gao, W., \& Sundberg, P. (2008). Phylogeny and classification of the old world Emberizini (Aves, Passeriformes). Molecular Phylogenetics and Evolution, 47(3), 960-973. doi:10.1016/j.ympev.2007.12.007

Avise, J. C. (2000). Phylogeography: The history and formation of species. Harvard University Press.

Baute, G. J., Owens, G. L., Bock, D. G., \& Rieseberg, L. H. (2016). Genome-wide genotyping-

Barrientos, A., Müller, S., Dey, R., Wienberg, J., \& Moraes, C. T. (2000). Cytochrome c oxidase assembly in primates is sensitive to small evolutionary variations in amino acid sequence. Molecular Biology and Evolution, 17(10), 1508-1519. https://doi.org/10.1093/oxfordjournals.molbev.a026250

Bolger, A. M., Lohse, M., \& Usadel, B. (2014). Trimmomatic: A flexible trimmer for Illumina sequence data. Bioinformatics, 30(15), 2114-2120. doi:10.1093/bioinformatics/btu170

Borge, T., Webster, M. T., Andersson, G., \& Saetre, G. (2005). Contrasting patterns of polymorphism and divergence on the $\mathrm{Z}$ chromosome and autosomes in two Ficedula flycatcher species. Genetics, 171(4), 1861-1873. doi:10.1534/genetics.105.045120 
652

653

654

655

656

657

658

659

660

661

662

663

664

665

666

667

668

669

670

671

672

673

674

675

676

677

678

679

680

681

682

683

684

685

Burton, R. S., \& Barreto, F. S. (2012). A disproportionate role for mtDNA in DobzhanskyMuller incompatibilities? Molecular Ecology, 21(20), 4942-4957.

doi:10.1111/mec.12006

Bryson, J., Robert W, De Oca, A. N., Jaeger, J. R., \& Riddle, B. R. (2010). Elucidation of cryptic diversity in a widespread Nearctic treefrog reveals episodes of mitochondrial gene capture as frogs diversified across a dynamic landscape. Evolution, 64(8), 23152330. https://doi.org/10.1111/j.1558-5646.2010.01014.x

Calvo, S. E., \& Mootha, V. K. (2010). The mitochondrial proteome and human disease. Annual Review of Genomics and Human Genetics, 11(1), 25-44.

doi:10.1146/annurev-genom-082509-141720

Coyne, J. A., \& Orr, H. A. (2004). Speciation. Sinauer Associates.

Cruickshank, T. E., \& Hahn, M. W. (2014). Reanalysis suggests that genomic islands of speciation are due to reduced diversity, not reduced gene flow. Molecular Ecology, 23(13), 3133-3157. doi:10.1111/mec.12796

Cutter, A. D., \& Payseur, B. A. (2013). Genomic signatures of selection at linked sites: Unifying the disparity among species. Nature Reviews. Genetics, 14(4), 262-274. doi: $10.1038 / \operatorname{nrg} 3425$

Danecek, P., Auton, A., Abecasis, G., Albers, C. A., Banks, E., DePristo, M. A., Handsaker, R. E., Lunter, G., Marth, G. T., Sherry, S. T., McVean, G., Durbin, R. \& 1000 Genomes Project Analysis Group. (2011). The variant call format and VCFtools. Bioinformatics, 27(15), 2156-2158. doi:10.1093/bioinformatics/btr330

Elshire, R. J., Glaubitz, J. C., Sun, Q., Poland, J. A., Kawamoto, K., Buckler, E. S., \& Mitchell, S. E. (2011). A robust, simple genotyping-by-sequencing (GBS) approach for high diversity species. PloS One, 6(5), e19379. doi:10.1371/journal.pone.0019379

Ernster, L., \& Schatz, G. (1981). Mitochondria: A historical review. The Journal of Cell Biology, 91(3), 227s-255s. doi:10.1083/jcb.91.3.227s 
Gascuel, O. (1997). BIONJ: An improved version of the NJ algorithm based on a simple model of sequence data. Molecular Biology and Evolution, 14(7), 685-695. doi:10.1093/oxfordjournals.molbev.a025808

Geraldes, A., Askelson, K. K., Nikelski, E., Doyle, F. I., Harrower, W. L., Winker, K., \& Irwin, D. E. (2019). Population genomic analyses reveal a highly differentiated and endangered genetic cluster of northern goshawks (Accipiter gentilis laingi) in Haida Gwaii. Evolutionary Applications, 12(4), 757-772. doi:10.1111/eva.12754

Gershoni, M., Levin, L., Ovadia, O., Toiw, Y., Shani, N., Dadon, S., Barzilai, N., Bergman, A., Atzmon, G., Wainstein, J., Tsur, A., Nijtmans, L. G. J., Glaser, B., \& Mishmar, D. (2014). Disrupting mitochondrial-nuclear coevolution affects OXPHOS complex I integrity and impacts human health. Genome Biology and Evolution, 6(10), 2665-2680.

Gershoni, M., Templeton, A. R., \& Mishmar, D. (2009). Mitochondrial bioenergetics as a major motive force of speciation. Bioessays, 31(6), 642-650. doi:10.1002/bies.200800139

Greber, B. J., \& Ban, N. (2016). Structure and function of the mitochondrial ribosome. Annual Review of Biochemistry, 85(1), 103-132. doi:10.1146/annurev-biochem-060815-014343

Harr, B. (2006). Genomic islands of differentiation between house mouse subspecies. Genome Research, 16(6), 730-737. doi:10.1101/gr.5045006

Hebert, P. D., Penton, E. H., Burns, J. M., Janzen, D. H., \& Hallwachs, W. (2004). Ten species in one: DNA barcoding reveals cryptic species in the neotropical skipper butterfly Astraptes fulgerator. Proceedings of the National Academy of Sciences, 101(41), 14812-

Hejase, H. A., Salman-Minkov, A., Campagna, L., Hubisz, M. J., Lovette, I. J., Gronau, I., \& Siepel, A. (2020). Genomic islands of differentiation in a rapid avian radiation have been driven by recent selective sweeps. Proceedings of the National Academy of Sciences, 117(48), 30554-30565. doi:10.1073/pnas.2015987117 
Hulsey, C. D., Bell, K. L., García-de-León, F. J., Nice, C. C., \& Meyer, A. (2016). Do relaxed selection and habitat temperature facilitate biased mitogenomic introgression in a narrowly endemic fish? Ecology and Evolution, 6(11), 3684- 3698. https://doi.org/10.1002/ece3.2121

Hulsey, C. D., \& García-de-León, F. J. (2013). Introgressive hybridization in a trophically polymorphic cichlid. Ecology and Evolution, 3(13), 4536-4547. https://doi.org/10.1002/ece3.841

International Chicken Genome Sequencing Consortium. (2004). Sequence and comparative analysis of the chicken genome provide unique perspectives on vertebrate evolution. Nature, 432(7018), 695-716. doi:10.1038/nature03154

Irwin, D. E. (2018). Sex chromosomes and speciation in birds and other ZW systems. Molecular Ecology, 27(19), 3831-3851. doi:10.1111/mec.14537

Irwin, D. E., Alcaide, M., Delmore, K. E., Irwin, J. H., \& Owens, G. L. (2016). Recurrent selection explains parallel evolution of genomic regions of high relative but low absolute differentiation in a ring species. Molecular Ecology, 25(18), 4488-4507. doi:10.1111/mec.13792

Irwin, D. E., Milá, B., Toews, D. P. L., Brelsford, A., Kenyon, H. L., Porter, A. N., Grossen, C., Delmore, K. E., Alcaide, M., \& Irwin, J. H. (2018). A comparison of genomic islands of differentiation across three young avian species pairs. Molecular Ecology, 27(23), 48394855. doi:10.1111/mec.14858

Irwin, D. E., Rubtsov, A. S., \& Panov, E. N. (2009). Mitochondrial introgression and replacement between yellowhammers (Emberiza citrinella) and pine buntings (Emberiza leucocephalos) (Aves: Passeriformes). Biological Journal of the Linnean Society, 98(2), 422-438. doi:10.1111/j.1095-8312.2009.01282.x

Kerr, K. C. R., Stoeckle, M. Y., Dove, C. J., Weigt, L. A., Francis, C. M., \& Hebert, P. D. N. (2007). Comprehensive DNA barcode coverage of North American birds. Molecular Ecology Notes, 7(4), 535-543. doi:10.1111/j.1471-8286.2007.01670.x

Kirkpatrick, M. (2010). How and why chromosome inversions evolve. PLoS Biology, 8(9), e1000501. doi:10.1371/journal.pbio.1000501 
Li, H., \& Durbin, R. (2009). Fast and accurate short read alignment with Burrows-Wheeler

762 transform. Bioinformatics, 25(14), 1754-1760. doi:10.1093/bioinformatics/btp324

Li, H., Handsaker, B., Wysoker, A., Fennell, T., Ruan, J., Homer, N., Marth, G., Abecasis, G., \& Durbin, R. (2009). The sequence alignment map format and SAMtools. Bioinformatics, 25(16), 2078-2079. doi:10.1093/bioinformatics/btp352

Lotz, C., Lin, A. J., Black, C. M., Zhang, J., Lau, E., Deng, N., Wang, Y., Zong, N. C., Choi, J. H., Xu, T., Liem, D. A., Korge, P., Weiss, J. N., Hermjakob, H., Yates, J. R., III., Apweiler, R., \& Ping, P. (2014). Characterization, design, and function of the mitochondrial proteome: From organs to organisms. Journal of Proteome Research, 13(2), 433-446. doi:10.1021/pr400539j

Lu, J., \& Wu, C. (2005). Weak selection revealed by the whole-genome comparison of the X chromosome and autosomes of human and chimpanzee. Proceedings of the National Academy of Sciences, 102(11), 4063-4067. doi:10.1073/pnas.0500436102

Lynch, M., Koskella, B., \& Schaack, S. (2006). Mutation pressure and the evolution of organelle genomic architecture. Science, 311(5768), 1727-1730. doi:10.1126/science.1118884

Mank, J. E., Nam, K., \& Ellegren, H. (2010). Faster-Z evolution is predominantly due to genetic drift. Molecular Biology and Evolution, 27(3), 661-670. doi:10.1093/molbev/msp282

Manthey, J. D., Geiger, M., \& Moyle, R. G. (2017). Relationships of morphological groups in the northern flicker superspecies complex (Colaptes auratus \& C. chrysoides). Systematics and Biodiversity, 15(3), 183-191.

McKenna, A., Hanna, M., Banks, E., Sivachenko, A., Cibulskis, K., Kernytsky, A., Garimella, K., Altshuler, D., Gabriel, S., Daley, M., \& DePristo, M. A. (2010). The genome analysis toolkit: A MapReduce framework for analyzing next-generation DNA sequencing data. Genome Research, 20(9), 1297-1303. doi:10.1101/gr.107524.110

Meisel, R. P., \& Connallon, T. (2013). The faster-X effect: Integrating theory and data. Trends in Genetics, 29(9), 537-544. doi:10.1016/j.tig.2013.05.009 
Moore, W. S. (1995). Inferring phylogenies from mtDNA variation: Mitochondrial-gene trees versus nuclear-gene trees. Evolution, 49(4), 718-726. doi:10.2307/2410325

Morales, H. E., Pavlova, A., Amos, N., Major, R., Kilian, A., Greening, C., \& Sunnucks, P. (2018). Concordant divergence of mitogenomes and a mitonuclear gene cluster in bird lineages inhabiting different climates. Nature Ecology \& Evolution, 2(8), 1258-1267. doi:10.1038/s41559-018-0606-3

Nadeau, N. J., Whibley, A., Jones, R. T., Davey, J. W., Dasmahapatra, K. K., Baxter, S. W., Quail, M. A., Joron, M., Ffrench-Constant, R. H., Blaxter, M. L., Mallet, J., \& Jiggins, C. D. (2012). Genomic islands of divergence in hybridizing Heliconius butterflies identified by large-scale targeted sequencing. Philosophical Transactions of the Royal Society B: Biological Sciences, 367(1587), 343-353. doi:10.1098/rstb.2011.0198

Osada, N., \& Akashi, H. (2012). Mitochondrial-nuclear interactions and accelerated compensatory evolution: Evidence from the primate cytochrome c oxidase complex. Molecular Biology and Evolution, 29(1), 337-346.

815 https://doi.org/10.1093/molbev/msr211

Panov, E. N., Rubtsov, A. S., \& Mordkovich, M. V. (2007). New data on the relationships between two species of buntings (Emberiza citrinella and E. leucocephalos) hybridizing in the areas of overlap of their ranges, Zoologicheskii Zhurnal, 86(11), 1362-1378.

Paradis, E., \& Schliep, K. (2019). ape 5.0: An environment for modern phylogenetics and evolutionary analyses in R. Bioinformatics, 35(3), 526-528.

Price, T. (2008). Speciation in birds. Roberts and Co. doi:10.1093/bioinformatics/bty633 
Rubtsov, A.S. 2007. Variability of songs of the yellowhammer (Emberiza citrinella) and pine bunting (Emberiza leucocephala) as an evidence of population structure and evolutionary history of the species, Zoologicheskii Zhurnal, 86(7), 863-876.

Rubtsov, A. S., \& Tarasov, V. V. (2017). Relations between the yellowhammer (Emberiza citrinella) and the pine bunting (Emberiza leucocephalos) in the forested steppe of the Trans-Urals. Biology Bulletin, 44(9), 1059-1072. doi:10.1134/S1062359017090114

Ruegg, K., Anderson, E. C., Boone, J., Pouls, J., \& Smith, T. B. (2014). A role for migrationlinked genes and genomic islands in divergence of a songbird. Molecular Ecology, 23(19), 4757-4769. doi:10.1111/mec.12842

Sackton, T. B., Corbett-Detig, R. B., Nagaraju, J., Vaishna, L., Arunkumar, K. P., \& Hartl, D. L. (2014). Positive selection drives faster-z evolution in silkmoths. Evolution, 68(8), 23312342. doi:10.1111/evo.12449

Sackton, T. B., Haney, R. A., \& Rand, D. M. (2003). Cytonuclear coadaptation in Drosophila: Disruption of cytochrome c oxidase activity in backcross genotypes. Evolution, 57(10), 2315-2325. https://doi.org/10.1111/j.0014-3820.2003.tb00243.x

Saraste, M. (1999). Oxidative phosphorylation at the fin de siècle. Science, 283(5407), 14881493. https://doi.org/10.1126/science.283.5407.1488

Scordato, E. S. C., Wilkins, M. R., Semenov, G., Rubtsov, A. S., Kane, N. C., \& Safran, R. J. (2017). Genomic variation across two barn swallow hybrid zones reveals traits associated with divergence in sympatry and allopatry. Molecular Ecology, 26(20), 56765691. https://doi.org/10.1111/mec.14276

Sloan, D. B., Havird, J. C., \& Sharbrough, J. (2017). The on-again, off-again relationship between mitochondrial genomes and species boundaries. Molecular Ecology, 26(8), 2212-2236. doi:10.1111/mec.13959

Smukowski, C. S., \& Noor, M. A. F. (2011). Recombination rate variation in closely related species. Heredity, 107(6), 496-508. doi:10.1038/hdy.2011.44 
Stacklies, W., Redestig, H., Scholz, M., Walther, D., \& Selbig, J. (2007). pcaMethods-a bioconductor package providing PCA methods for incomplete data. Bioinformatics, 23(9), 1164-1167. doi:10.1093/bioinformatics/btm069

Tajima, F. (1989). Statistical method for testing the neutral mutation hypothesis by DNA polymorphism. Genetics, 123(3), 585-595.

Thornton, K., \& Long, M. (2002). Rapid divergence of gene duplicates on the Drosophila melanogaster X chromosome. Molecular Biology and Evolution, 19(6), 918-925. doi:10.1093/oxfordjournals.molbev.a004149

Toews, D. P. L., \& Brelsford, A. (2012). The biogeography of mitochondrial and nuclear discordance in animals. Molecular Ecology, 21(16), 3907-3930. doi:10.1111/j.1365294X.2012.05664.X

Turner, S. (2018). qqman: An R package for visualizing GWAS results using Q-Q and Manhattan plots. Journal of Open Source Software, 3(25), 731. doi:10.21105/joss.00731

Wang, S., Ore, M. J., Mikkelsen, E. K., Lee-Yaw, J., Toews, D. P. L., Rohwer, S., \& Irwin, D. (2021). Signatures of mitonuclear coevolution in a warbler species complex. Nature Communications, in press. doi:10.1038/s41467-021-24586-8

Warren, W. C., Clayton, D. F., Ellegren, H., Arnold, A. P., Hillier, L. W.,Künstner, A., Searle, S., White, S., Vilella, A. J., Fairley, S., Heger, A., Kong, L., Ponting, C. P., Jarvis, E. D., Mello, C. V., Minx, P., Lovell, P., Velho, T. A. F., Ferris, M., ... Wilson, R. K. (2010). The genome of a songbird. Nature, 464(7289), 757-762. doi:10.1038/nature08819

Weir, B. S., \& Cockerham, C. C. (1984). Estimating F-statistics for the analysis of population structure. Evolution, 38(6), 1358-1370. doi:10.1111/j.1558-5646.1984.tb05657.x

Wu, C. (2001). The genic view of the process of speciation. Journal of Evolutionary Biology, 14(6), 851-865. doi:10.1046/j.1420-9101.2001.00335.x

Yannic, G., Dubey, S., Hausser, J., \& Basset, P. (2010). Additional data for nuclear DNA give new insights into the phylogenetic position of Sorex granarius within the Sorex araneus 
bioRxiv preprint doi: https://doi.org/101101/2021.08 08 455564; this version posted August 8, 2021. The copyright holder for this preprint (which was not certified by peer review) is the author/funder, who has granted bioRxiv a license to display the preprint in perpetuity. It is made available under aCC-BY-NC-ND 4.0 International license.

902

903

904

905

906

907 group. Molecular Phylogenetics and Evolution, 57(3), 1062-

1071. https://doi.org/10.1016/j.ympev.2010.09.015

Zink, R. M., \& Barrowclough, G. F. (2008). Mitochondrial DNA under siege in avian phylogeography. Molecular Ecology, 17(9), 2107-2121. doi:10.1111/j.1365294X.2008.03 


\section{Tables}

Table 1. Geographical locations and sample sizes of the sites included in this study. Sampling locations may include multiple sites that appeared too close together to be shown in detail in Figure 1A. Full details for the sites included in each sampling locations can be found in Supplementary Table 1. The sampling location numbers that appear in the "Sampling Location" column correspond to those that appear in red in Figure 1A. The "Sample Size" columns describes the total number of samples collected from a particular location.

\begin{tabular}{|c|c|c|c|c|}
\hline $\begin{array}{l}\text { Sampling } \\
\text { Location }\end{array}$ & $\begin{array}{l}\text { Latitude } \\
\left({ }^{\circ} \mathrm{N}\right)\end{array}$ & Longitude $\left({ }^{\circ} \mathrm{E}\right)$ & $\begin{array}{l}\text { E. citrinella } \\
\text { sample size }\end{array}$ & $\begin{array}{l}\text { E. leucocephalos } \\
\text { sample size }\end{array}$ \\
\hline 1 & 57.99 & 12.49 & 1 & 0 \\
\hline 2 & 59.81 & 17.05 & 1 & 0 \\
\hline 3 & 51.71 & 18.61 & 1 & 0 \\
\hline 4 & 55.28 & 20.97 & 5 & 0 \\
\hline 5 & 65.86 & 21.48 & 2 & 0 \\
\hline 6 & 51.38 & 35.84 & 3 & 0 \\
\hline 7 & 55.97 & 38.50 & 18 & 0 \\
\hline 8 & 61.45 & 38.67 & 12 & 0 \\
\hline 9 & 43.54 & 40.47 & 1 & 0 \\
\hline 10 & 65.85 & 44.24 & 1 & 0 \\
\hline 11 & 58.33 & 44.76 & 1 & 0 \\
\hline 12 & 51.20 & 57.27 & 7 & 0 \\
\hline 13 & 49.64 & 110.17 & 0 & 2 \\
\hline 14 & 50.66 & 115.09 & 0 & 17 \\
\hline 15 & 51.12 & 118.56 & 0 & 15 \\
\hline \multirow[t]{2}{*}{16} & 50.56 & 143.08 & 0 & 8 \\
\hline & & Total & 53 & 42 \\
\hline
\end{tabular}


Table 2. Summary statistics calculated while conducting mitonuclear co-introgression analysis. A total of 7187 windows, each of $2000 \mathrm{bp}$ of obtained sequence, were considered when determining introgression windows. A total of 134 mitonuclear genes were investigated for signals of co-introgression. "*” indicates a significant $p$-value.

\begin{tabular}{lllll}
\hline \hline Species & $\begin{array}{l}\text { \# of introgression } \\
\text { windows identified }\end{array}$ & $\begin{array}{l}\text { \% of mitonuclear genes } \\
\text { appearing in introgression } \\
\text { windows }\end{array}$ & $\begin{array}{l}\text { Binomial test } \\
\text { p-value }\end{array}$ & $\begin{array}{l}\text { Fisher's Exact test } \\
\text { p-value }\end{array}$ \\
\hline Yellowhammer & 244 & 6.7 & $0.04952^{*}$ & 0.1311 \\
Pine bunting & 222 & 3.0 & 1 & 1 \\
\hline
\end{tabular}


Table 3. Identities, chromosomal locations, windowed Tajima's D values and functions of mitonuclear genes that appeared within 244 yellowhammer introgression windows. In the "Mitonuclear Gene Function" column, ETC stands for "Electron Transport Chain". Mitonuclear gene names are written as they appear in Hill (2019).

\begin{tabular}{|c|c|c|c|}
\hline Mitonuclear Gene & $\begin{array}{l}\text { Chromosome where } \\
\text { mitonuclear gene is found }\end{array}$ & $\begin{array}{l}\text { Windowed Tajima's D } \\
\text { Value }\end{array}$ & Mitonuclear Gene Function \\
\hline APOPT1 & 5 & -2.207 & Assembly factor/ancillary protein for ETC complex IV \\
\hline COX5A & 10 & -2.420 & Structural subunit of ETC complex IV \\
\hline COX17 & 1 & -2.509 & Assembly factor/ancillary protein for ETC complex IV \\
\hline MRPL1 & 4 & -2.207 & Mitochondrial large ribosomal subunit protein \\
\hline MRPL27 & 18 & -2.214 & Mitochondrial large ribosomal subunit protein \\
\hline MRPL32 & 2 & -2.306 & Mitochondrial large ribosomal subunit protein \\
\hline NDUFC1 & 4 & -2.399 & Structural subunit of ETC complex I \\
\hline $\mathrm{mtSSB}$ & $1 \mathrm{~A}$ & -2.362 & Single stranded DNA-binding protein \\
\hline UQCR11 & 28 & -2.499 & Structural subunit of ETC complex III \\
\hline
\end{tabular}


Table 4. Identities, chromosomal locations, windowed Tajima's D values and functions of mitonuclear genes that appeared within 222 pine bunting introgression windows. In the "Mitonuclear Gene Function" column, ETC stands for "Electron Transport Chain". Mitonuclear gene names are written as they appear in Hill (2019).

\begin{tabular}{llll}
\hline \hline Mitonuclear Gene & $\begin{array}{l}\text { Chromosome where } \\
\text { mitonuclear gene is found }\end{array}$ & $\begin{array}{l}\text { Windowed Tajima's D } \\
\text { Value }\end{array}$ & Mitonuclear Gene Function \\
\hline ATP5H5I & 18 & -2.601 & Structural subunit of ETC complex V \\
COX5A & 10 & -2.545 & Mitochondrial large ribosomal subunit protein \\
MRPL2 & 3 & -2.247 & Structural subunit of ETC complex I \\
\hline
\end{tabular}




\section{Figures}

A.

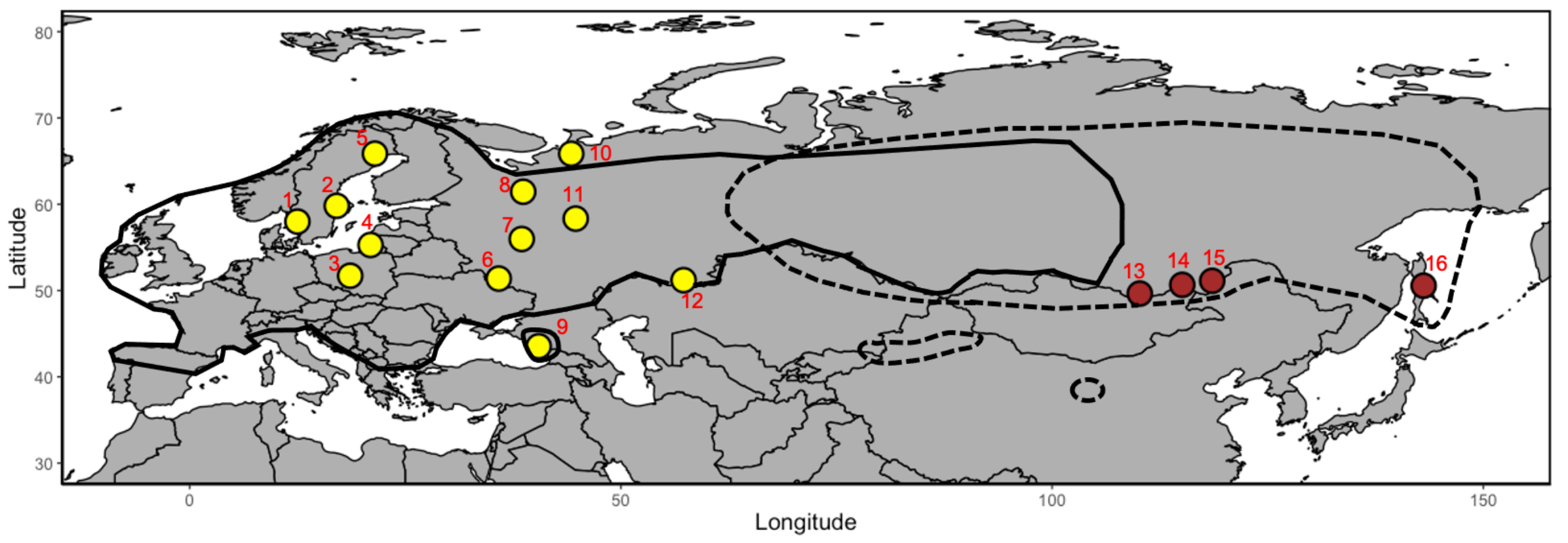

E. citrinella

$\bigcirc$

E. leucocephalos

B.

Emberiza citrinella

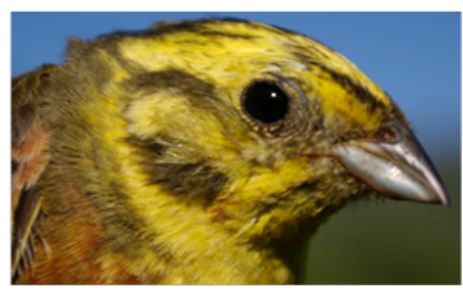

Pure Citrinella (PC)

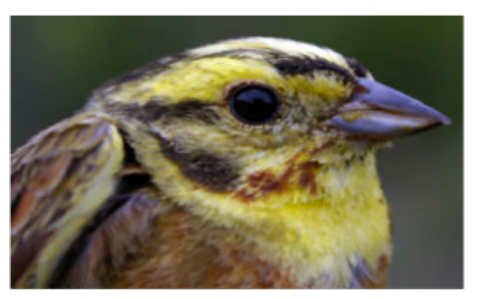

Almost Citrinella (SC)

\section{Emberiza leucocephalos}

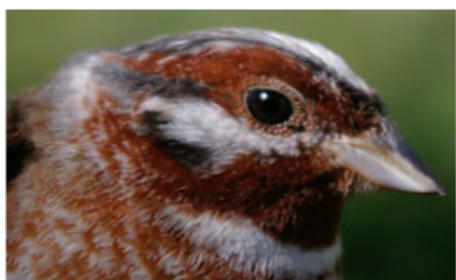

Pure Leucocephalos (PL)

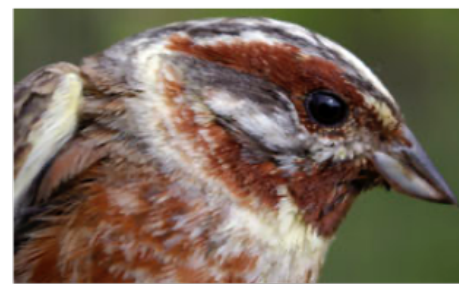

Almost Leucocephalos (SL) 
Figure 1. A) Map of sampling locations included in this study. Red numbers accompanying each location correspond to the sampling location numbers appearing in Table 1 which also describes sample sizes. Sampling locations may include multiple sites that appeared too close together to be shown in detail in this figure. Full details for the sites included in each sampling location can be found in Supplementary Table 1. Sampling location points are coloured based on the taxon caught in each area: yellowhammer (Emberiza citrinella; yellow) and pine bunting (Emberiza leucocephalos; brown). The solid black line indicates the geographic range of the yellowhammer and the dashed black line indicates the geographic range of the pine bunting as described in Irwin et al. (2009). B) Photos of plumage variation between yellowhammers and pine buntings. Each photo represents one of four phenotypic classes: PC, SC, PL and SL. Individuals with a PC and SC phenotypic class were grouped together as Emberiza citrinella and individuals with a PL and SL phenotypic class were grouped together as Emberiza leucocephalos. All photos are credited to Dr. Alexander Rubtsov. 


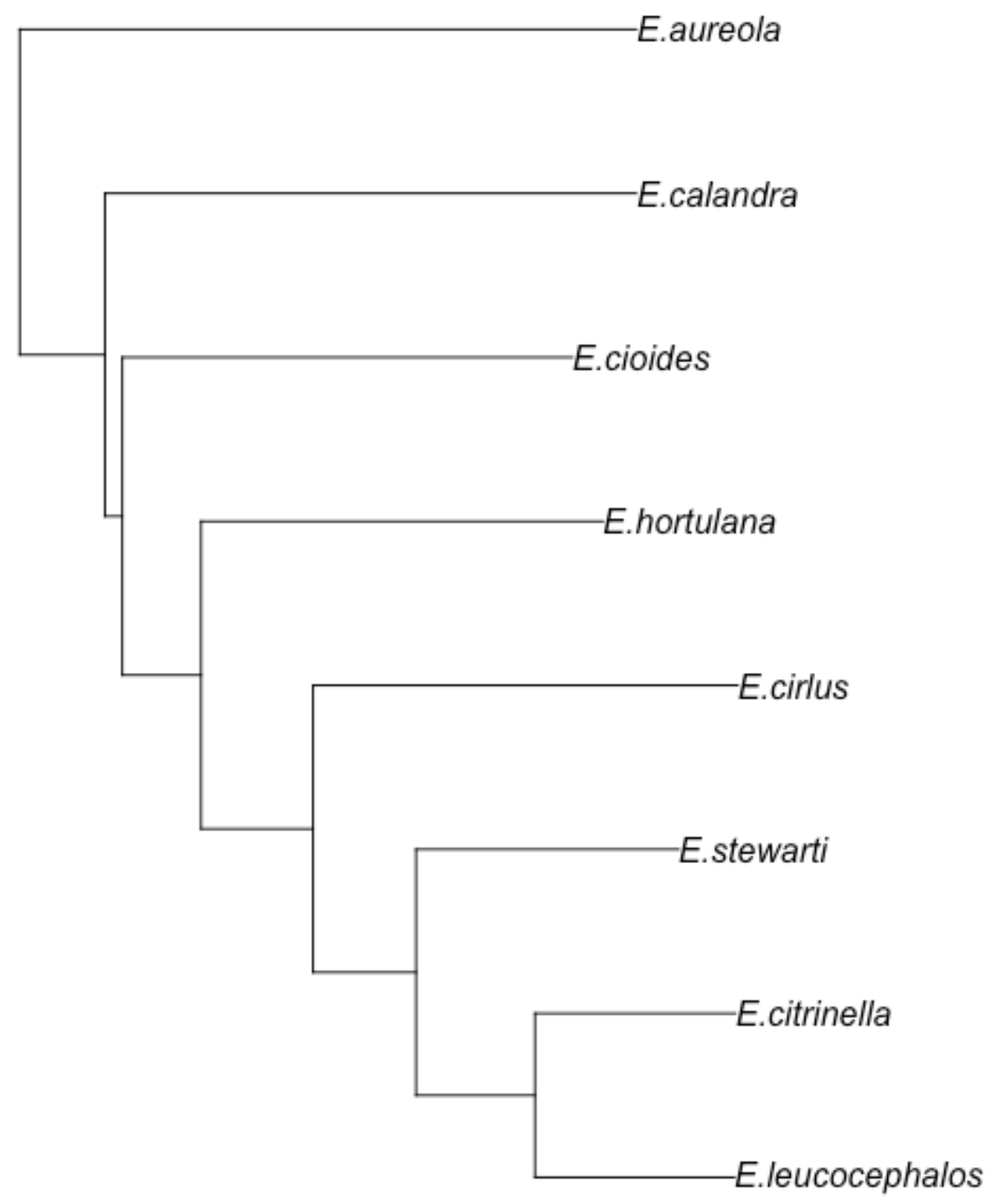

Figure 2. Unrooted neighbour-joining tree of Emberizidae species constructed based on average absolute between-population nucleotide diversity $\left(\pi_{B}\right)$. Sample sizes for each species are as follows: $E$. aureola $=1, E$. calandra $=1, E$. cioides $=1, E$. hortulana $=1, E$. cirlus $=6$, E. stewarti $=4$, E. citrinella $=53$ and E. leucocephalos $=42$. 


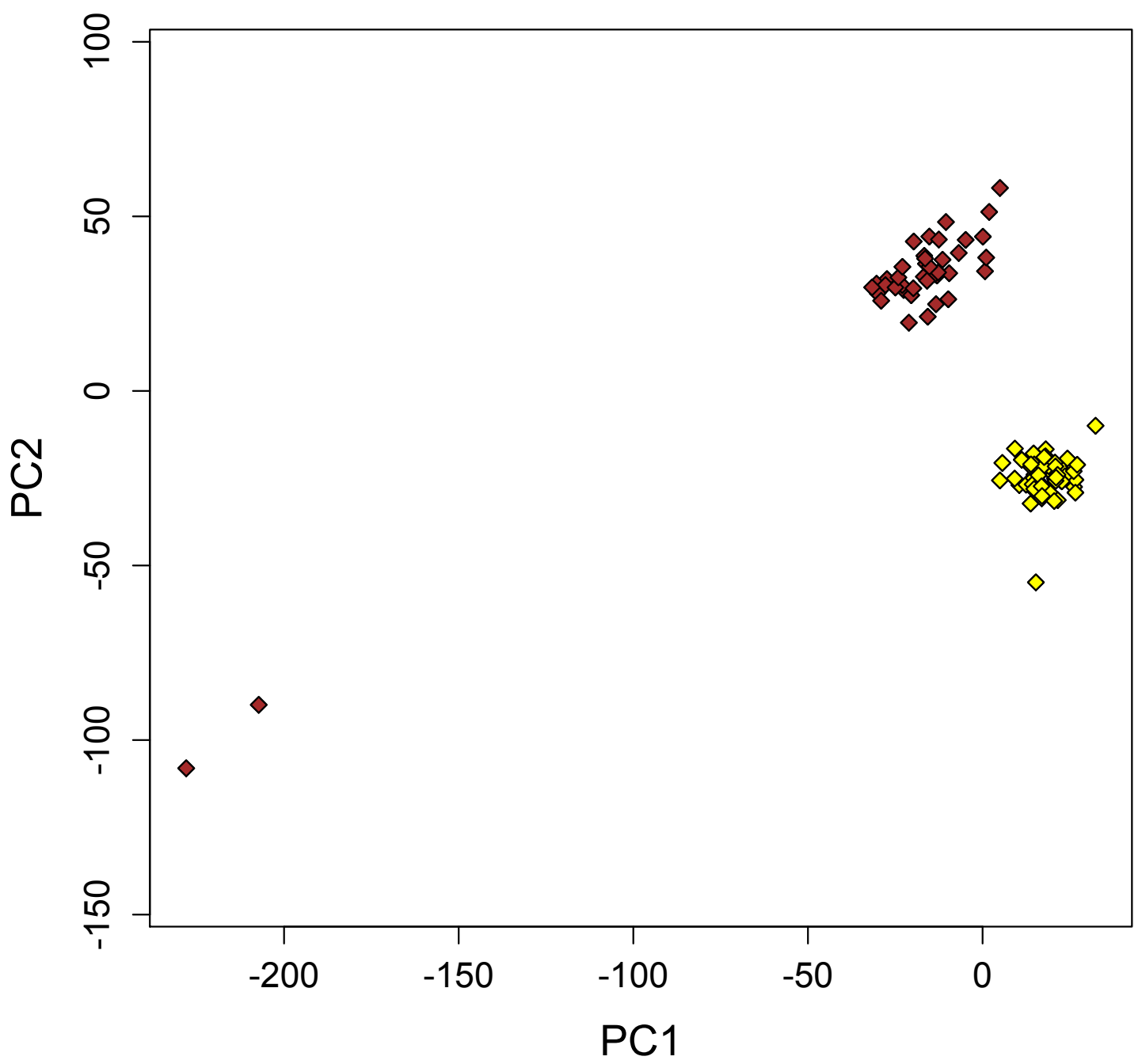

Figure 3. PCA of genetic variation between allopatric yellowhammers (yellow; $n=53$ ) and allopatric pine buntings (brown; $\mathrm{n}=42$ ), based on 374,780 genome-wide SNPs. PC1 and PC2 explain 3.6\% and $2.9 \%$, respectively, of the variation among individuals. 


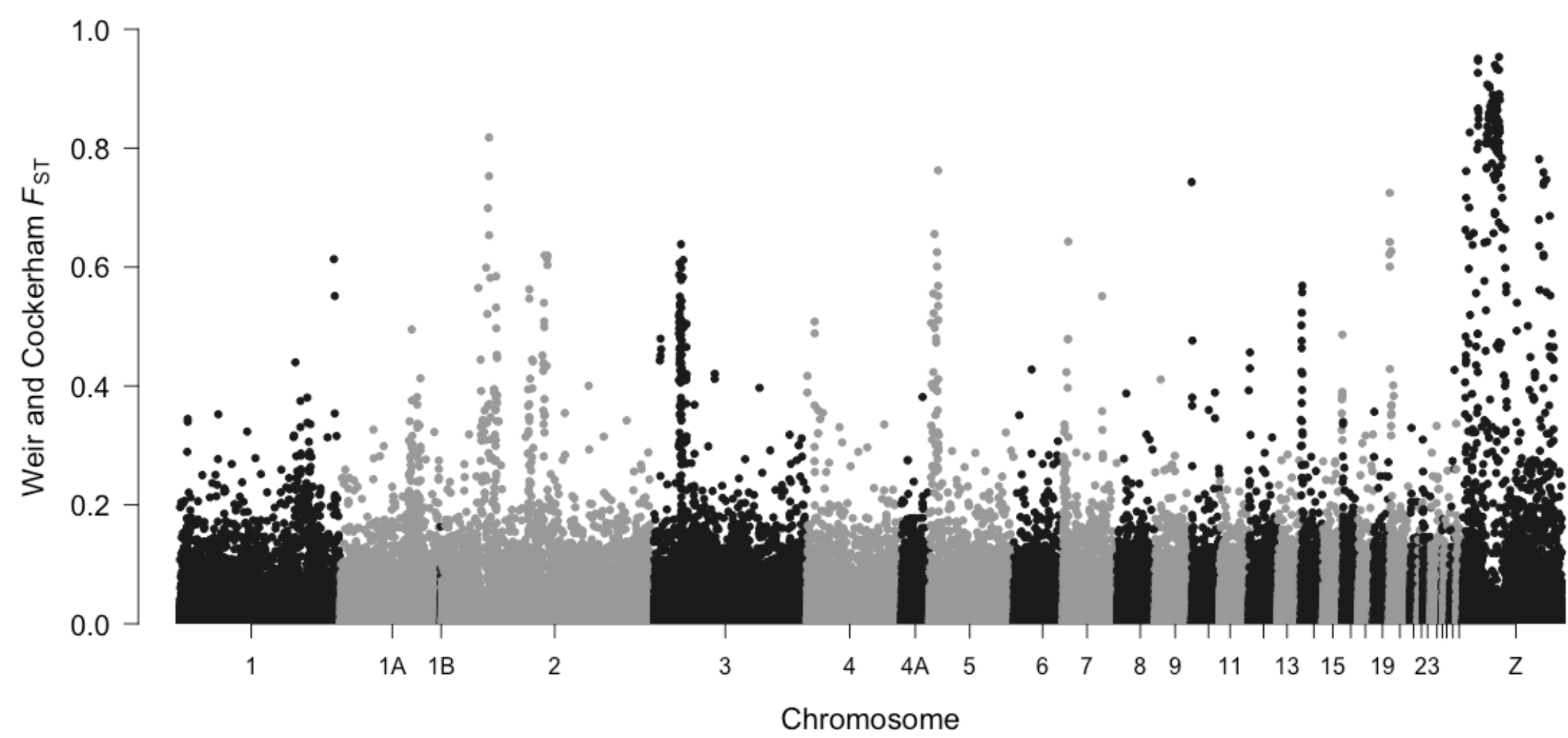

Figure 4. Relative differentiation $\left(F_{\mathrm{ST}}\right)$ of 349,807 genome-wide SNPs identified between allopatric yellowhammers $(\mathrm{n}=53)$ and allopatric pine buntings $(\mathrm{n}=42)$, with chromosomes represented with alternating black and grey. Narrow regions of elevated differentiation can be seen on many autosomes, and there are broad regions of high differentiation on the $\mathrm{Z}$ chromosome. 

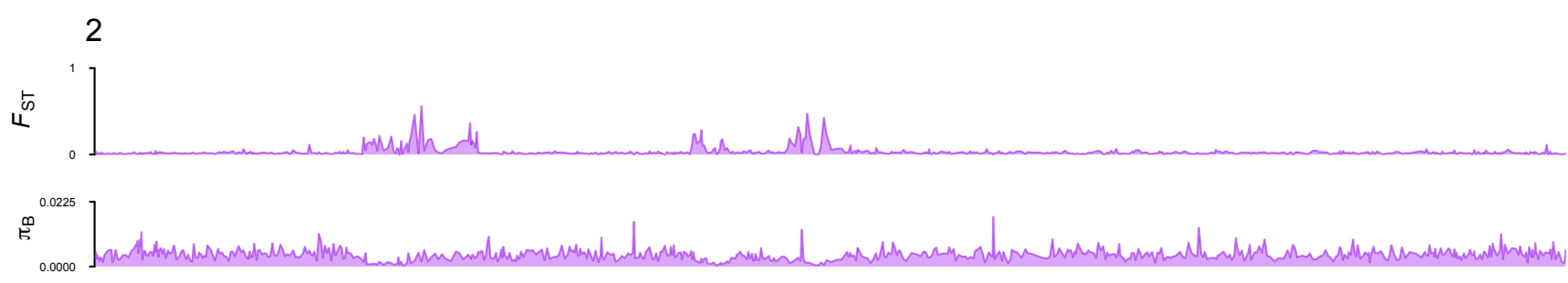

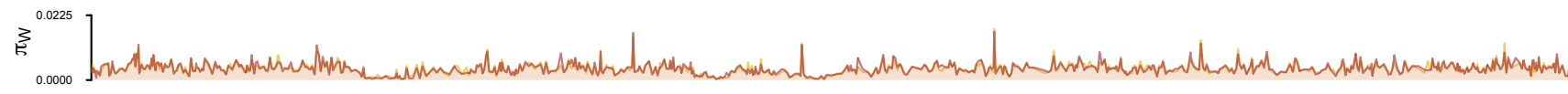

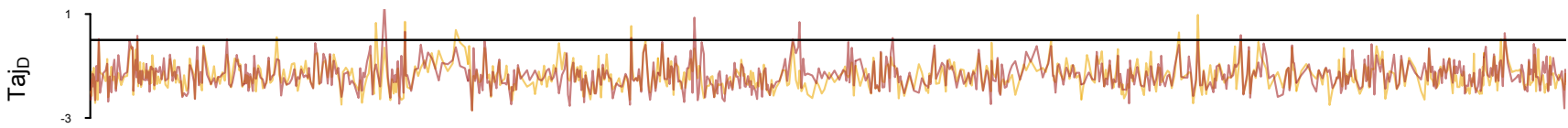

5
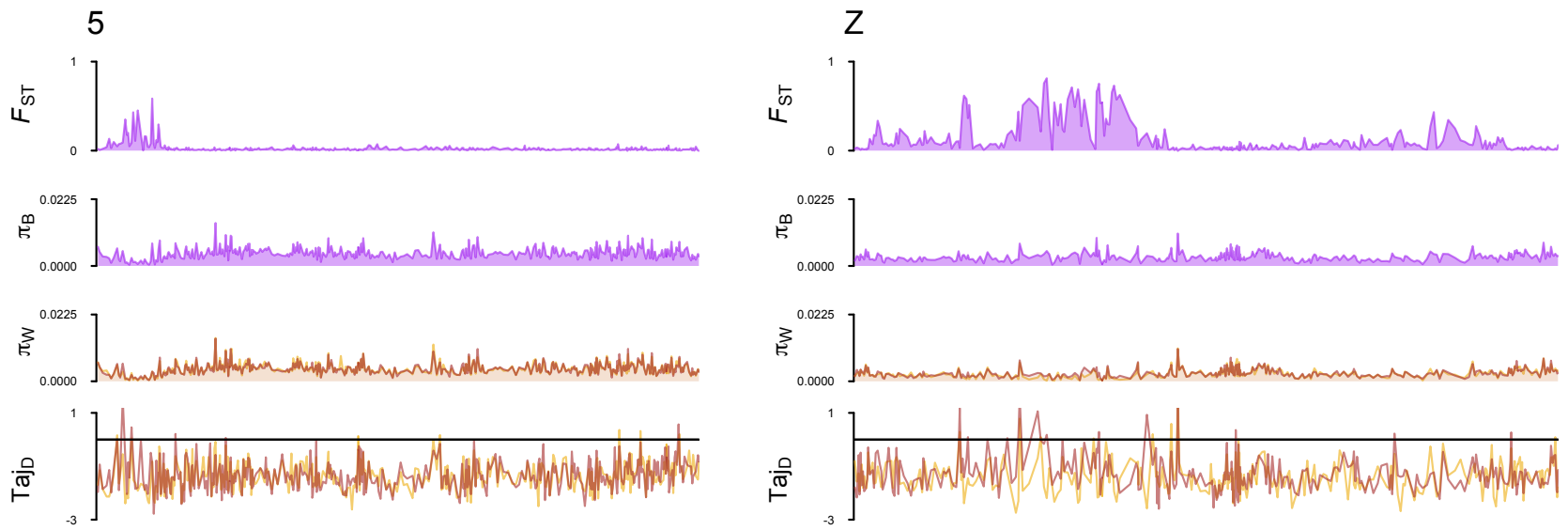

Figure 5. Patterns of genetic variation comparing allopatric yellowhammers $(n=53)$ and allopatric pine buntings $(\mathrm{n}=42)$ across three chromosomes $(2,5$ and $\mathrm{Z})$. Relative nucleotide differentiation $\left(F_{\mathrm{ST}}\right)$, absolute between-population nucleotide diversity $\left(\pi_{B}\right)$, absolute withinpopulation nucleotide diversity $\left(\pi_{W}\right)$ and Tajima's D (TajD) are shown as 2000 bp windowed averages across each chromosome. $F_{\mathrm{ST}}$ and $\pi_{B}$ are shown as purple lines to indicate that values were calculated as a comparison between allopatric yellowhammers and pine buntings. $\pi_{W}$ and Taj $_{\mathrm{D}}$ are shown as two separate lines (yellow $=$ yellowhammers, brown $=$ pine buntings) to indicate that values were calculated separately for each population. 


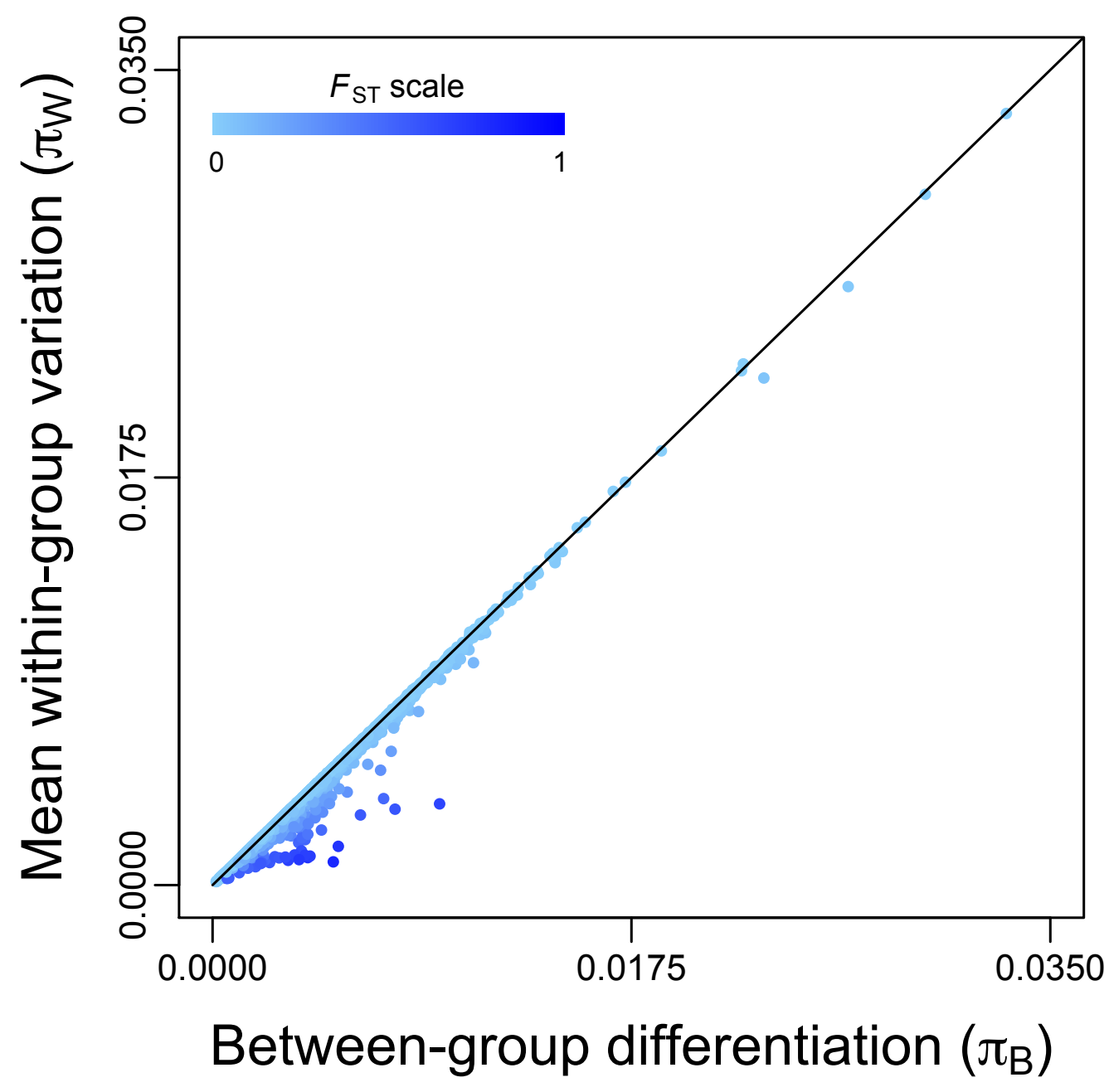

Figure 6. Mean absolute within-group nucleotide diversity $\left(\pi_{W}\right)$ of allopatric yellowhammers $(\mathrm{n}$ $=53)$ and allopatric pine buntings $(\mathrm{n}=42)$ plotted against absolute between-group nucleotide diversity $\left(\pi_{B}\right)$. Each dot represents the average value taken from a $2000 \mathrm{bp}$ window of sequenced data across the nuclear genome. The black line indicates where mean within-group nucleotide diversity equals between-group nucleotide diversity. Increasing values of relative differentiation $\left(F_{\mathrm{ST}}\right)$ calculated for each window are shown in darker shades of blue. 


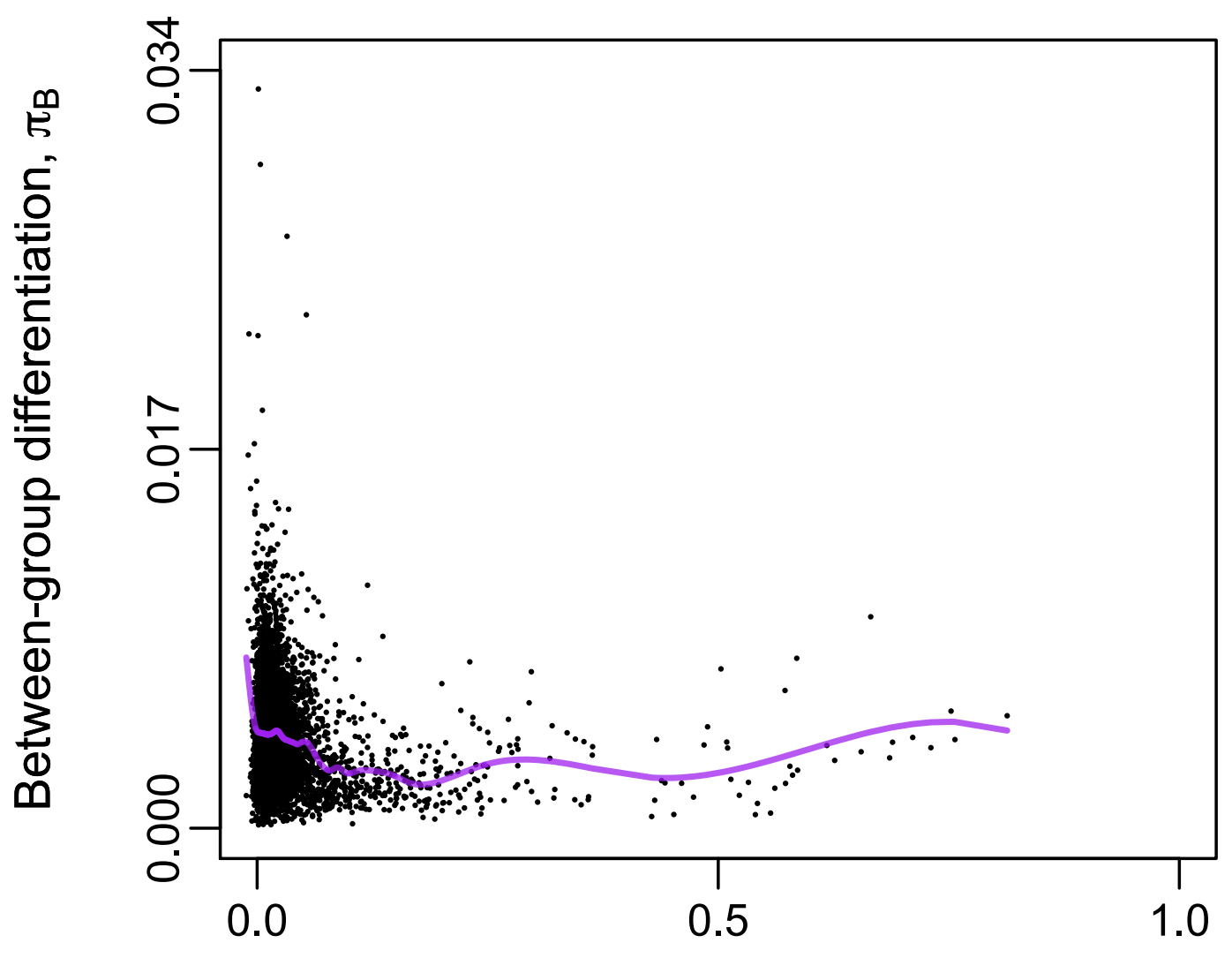

\section{Relative differentiation, $F_{\mathrm{ST}}$}

Figure 7. Association between relative differentiation $\left(F_{\mathrm{ST}}\right)$ and absolute between-group nucleotide diversity $\left(\pi_{B}\right)$ of allopatric yellowhammers $(\mathrm{n}=53)$ and allopatric pine buntings $(\mathrm{n}=$ 42). Each black dot represents average values calculated from a $2000 \mathrm{bp}$ window of sequenced data. A cubic spline fit between the variables is shown as a purple line. 